\title{
Memoryless Determinacy of Finite Parity Games: Another Simple Proof 败, 该访
}

\author{
Serge Haddad \\ LSV, ENS Paris-Saclay \& CNRS \& Inria, Université Paris-Saclay \\ 61, avenue du Président Wilson 94235 CACHAN Cedex, France
}

\begin{abstract}
Memoryless determinacy of (infinite) parity games is an important result with numerous applications. It was first independently established by Emerson and Jutla [1] and Mostowski [2] but their proofs involve elaborate developments. The elegant and simpler proof of Zielonka [3] still requires a nested induction on the finite number of priorities and on ordinals for sets of vertices. There are other proofs for finite games like the one of Björklund, Sandberg and Vorobyovin [4] that relies on relating infinite and finite duration games. We present here another simple proof that finite parity games are determined with memoryless strategies using induction on the number of relevant states. The closest proof that relies on induction over non absorbing states is the one of Grädel [5]. However instead of focusing on a single appropriate vertex for induction as we do here, he considers two reduced games per vertex, for all the vertices of the game. The idea of reasoning about a single state has been inspired to me by the analysis of finite stochastic priority games by Karelovic and Zielonka [6].
\end{abstract}

Keywords: Game Theory, Determinacy, Memoryless Strategy

\footnotetext{
This work has been supported by ERC project EQualIS (FP7-308087).

Whe author thanks the participants of the working group Vasco-Mexico of LSV that have suggested useful improvements for presentation of the proof.

Email address: serge.haddad@lsv.fr (Serge Haddad)
} 


\section{Parity Games}

A parity game is a graph where vertices have integer priorities. In addition every vertex is owned by one of the two players and has at least one successor.

Definition 1. A parity game $G=(V, E, \pi)$ is defined by:

- A finite set of vertices $V=V_{0} \uplus V_{1}$;

- A set of edges $E \subseteq V \times V$ such that for all $v \in V$ there exists $(v, w) \in E$;

- A priority mapping $\pi: V \rightarrow \mathbb{N}$.

The set of players is $\{0,1\}$. The parity of an integer $p$ (i.e. $p \bmod 2$ ) will be denoted more concisely by $\hat{p}$. Given a vertex $v$, player $\mathrm{P}_{v}$ is the owner of $v: \mathrm{P}_{v}=0$ if and only if $v \in V_{0}$.

Example 1. Figure 1 describes a parity game. The vertices owned by Player 0 (resp. 1) are represented by circles (resp. squares). The priorities are noted inside vertices.

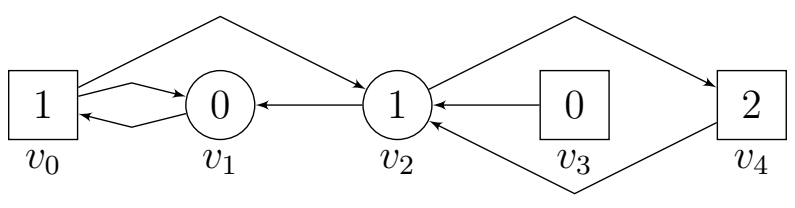

Figure 1: A parity game.

A play is a non empty finite or infinite sequence $v_{0} v_{1} \ldots$ such for all $i$ if $v_{i+1}$ is defined then $\left(v_{i}, v_{i+1}\right) \in E$. Consider an infinite play $\rho \in V^{\omega}$. Then $\max (\rho)$ is the maximal priority occurring infinitely often along the vertices of $\rho$. The winner of the play $\rho$ is Player $\max (\rho)$.

Example 2. $\rho=v_{4} v_{2}\left(v_{1} v_{0}\right)^{\omega}$ is a play of the game of Figure 1 winning for Player 1.

A strategy $\sigma$ for player $\mathrm{P}$ is a mapping from finite plays ending in $V_{\mathrm{P}}$ to $V$ such that $\left(v_{n}, \sigma\left(v_{0} \ldots v_{n}\right)\right) \in E$. An infinite play $\rho=v_{0} v_{1} \ldots$ is $\sigma$-compatible if for all $v_{n} \in V_{\mathrm{P}}, v_{n+1}=\sigma\left(v_{0} \ldots v_{n}\right)$. A strategy is memoryless if it only depends on the last state of the play. 
Definition 2. Let $G$ be a parity game. Then the winning set $\mathrm{W}_{\mathrm{P}}(G)$ of a player $\mathrm{P}$ is the set of vertices $v$ for which there exists a strategy $\sigma$ of $\mathrm{P}$ such that all $\sigma$-compatible (infinite) plays starting in $v$ are winning for $\mathrm{P}$.

Example 3. $\mathbf{W}_{1}(G)=\left\{v_{0}, v_{1}\right\}$ with Player 1 choosing the edge $\left(v_{0}, v_{1}\right)$ in $v_{0}$ while $\mathbf{W}_{0}(G)=\left\{v_{2}, v_{3}, v_{4}\right\}$ with Player 0 choosing the edge $\left(v_{2}, v_{4}\right)$ in $v_{2}$. Thus these winning strategies are memoryless.

A vertex $v$ of $G$ is absorbing if $\{v\}=\{w \mid(v, w) \in E\}$. A vertex $v$ of $G$ is vanishing if $\{w \mid(w, v) \in E\}=\emptyset$. A vertex is relevant if it is neither absorbing nor vanishing. Given a game, we split $V$ in the set of absorbing states $V_{\mathrm{a}}$, the set of vanishing states $V_{\mathrm{v}}$ and the set of relevant states $V_{\mathrm{r}}$.

\section{Memoryless determinacy}

Theorem 1. For all parity games $G, V=\mathrm{W}_{0}(G) \uplus \mathrm{W}_{1}(G)$ and furthermore:

- The corresponding strategies $\sigma_{0}$ and $\sigma_{1}$ are memoryless;

- For all $\mathrm{P} \in\{0,1\}$, a $\sigma_{\mathrm{P}}$-compatible play starting in $\mathrm{W}_{\mathrm{P}}(G)$ never leaves $\mathrm{W}_{\mathrm{P}}(G)$.

Proof. The proof is done by induction on the size of $\left|V_{\mathrm{r}}\right|$.

- When $\left|V_{\mathrm{r}}\right|=\emptyset$, by definition of $V_{\mathrm{v}}$ and $V_{\mathrm{a}}$ all strategies are memoryless and the winning sets are defined as follows.

$$
\begin{array}{rrr}
\mathrm{W}_{\mathrm{P}}(G)= & \left\{v \in V_{\mathrm{a}} \mid \widehat{\pi(v)}=\mathrm{P}\right\} \\
\cup & \left\{v \in V_{\mathrm{v}} \cap V_{\mathrm{P}} \mid \exists(v, w) \in E \widehat{\pi(w)}=\mathrm{P}\right\} \\
\cup & \left\{v \in V_{\mathrm{v}} \backslash V_{\mathrm{P}} \mid \forall(v, w) \in E \widehat{\pi(w)}=\mathrm{P}\right\}
\end{array}
$$

- Otherwise pick some $v \in V_{\mathrm{r}}$ with maximal priority, denoted $p$. Let $G^{+}$be the game obtained from $G$ by adding an absorbing state $\tilde{v}$ with priority and owner of $v$ and then redirecting incoming edges of $v$ to $\tilde{v}$ (see Figure 2).

- $V_{\mathrm{r}}^{+}=V_{\mathrm{r}} \backslash\{v\}, V_{\mathrm{a}}^{+}=V_{\mathrm{a}}^{+} \cup\{\tilde{v}\}$ and $V_{\mathrm{v}}^{+}=V_{\mathrm{v}} \cup\{v\}$;

- The owners of vertices are unchanged and $\mathrm{P}_{\tilde{v}}=\mathrm{P}_{v}$.

- For all $\left(u, u^{\prime}\right) \in E \cap V \times V \backslash\{v\},\left(u, u^{\prime}\right) \in E^{+}$; 
- $(\tilde{v}, \tilde{v}) \in E^{+}$and for all $(u, v) \in E,(u, \tilde{v}) \in E^{+}$;

- The priorities are unchanged and $\pi(\tilde{v})=p$.

The graph $G^{+}$fulfills: $\left|V_{\mathrm{r}}^{+}\right|=\left|V_{\mathrm{r}}\right|-1$. So the induction applies. Given a player $\mathrm{P}$, let $\sigma_{\mathrm{P}}^{+}$be a memoryless winning strategy of $\mathrm{P}$ in $G^{+}$.
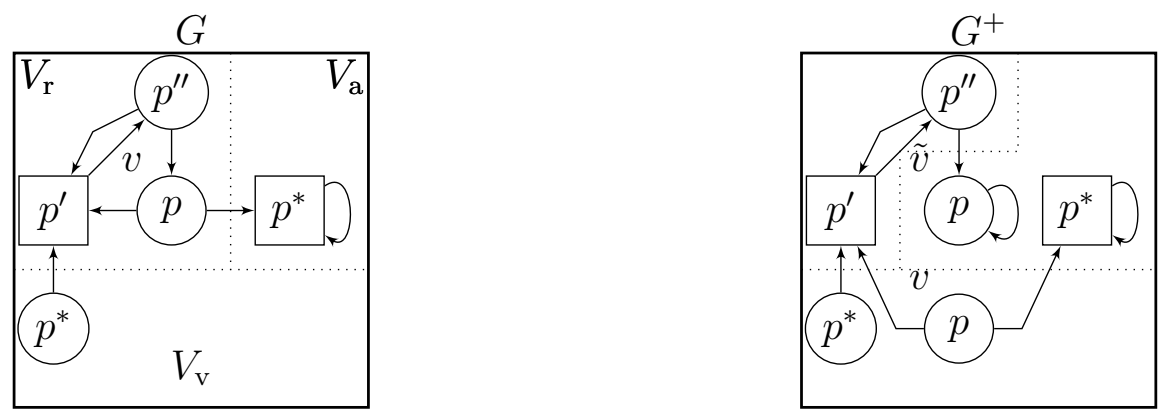

Figure 2: From $G$ to $G^{+}$

We claim that $\mathrm{W}_{1-\hat{p}}\left(G^{+}\right) \subseteq \mathrm{W}_{1-\hat{p}}(G)$. Consider in $G$, a $\sigma_{1-\hat{p}^{+}}^{+}$-compatible play $\rho$ starting in $\mathrm{W}_{1-\hat{p}}\left(G^{+}\right)$. Then it will never visit $v$ except possibly initially. So $\rho$ is a $\sigma_{1-\hat{p}^{+}}^{+}$-compatible play in $G^{+}$starting in $\mathrm{W}_{1-\hat{p}}\left(G^{+}\right)$. Hence $\rho$ is winning for Player $1-\hat{p}$.

- Case $v \in \mathrm{W}_{\hat{p}}\left(G^{+}\right)$. Consider $\rho$ a $\sigma_{\hat{p}}^{+}$-compatible play in $G$ starting in $\mathrm{W}_{\hat{p}}\left(G^{+}\right) \backslash\{\tilde{v}\}$. By definition of $G^{+}$and the property of strategy $\sigma_{\hat{p}}^{+}$, it will never leave $\mathrm{W}_{\hat{p}}\left(G^{+}\right)$.

- Either $\rho$ visits finitely often $v$ and then some suffix is a $\sigma_{\hat{p}}^{+}$-compatible play of $G^{+}$starting in $\mathrm{W}_{\hat{p}}\left(G^{+}\right)$. So this suffix and $\rho$ are winning for Player $\hat{p}$.

- Or $\rho$ visits infinitely often $v$ and thus never visits $V_{\mathrm{a}}$. Also observe that it may only initially visit $V_{\mathrm{v}}$. So the maximal priority of $\rho$ is $p$ and thus is winning for Player $\hat{p}$.

So $\mathrm{W}_{\hat{p}}\left(G^{+}\right) \backslash\{\tilde{v}\} \subseteq \mathrm{W}_{\hat{p}}(G)$.

Since $\mathrm{W}_{1-\hat{p}}\left(G^{+}\right) \subseteq \mathrm{W}_{1-\hat{p}}(G)$ memoryless determinacy is established.

- Case $v \in \mathrm{W}_{1-\hat{p}}\left(G^{+}\right)$. Let us build an auxiliary game $G^{-}$from $G$ by making $v$ absorbing and changing its priority to $p+1$ (see Figure 3): 

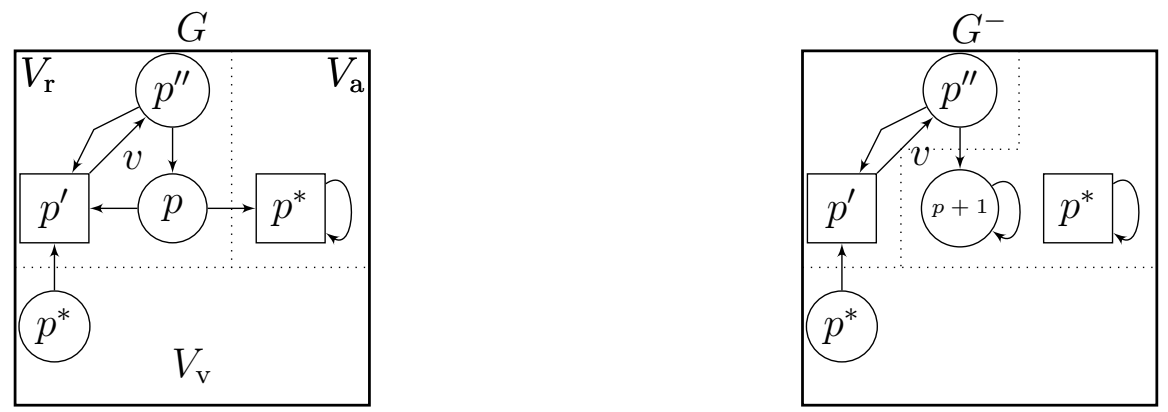

Figure 3: From $G$ to $G^{-}$

The graph $G^{-}$fulfills: $\left|V_{\mathrm{r}}^{-}\right|<\left|V_{\mathrm{r}}\right|$. Thus the induction applies. We claim that for all player $\mathrm{P}, \mathrm{W}_{\mathrm{P}}(G)=\mathrm{W}_{\mathrm{P}}\left(G^{-}\right)$. Given a player $\mathrm{P}$, let $\sigma_{\mathrm{P}}^{-}$be a memoryless winning strategy of $\mathrm{P}$ in $G^{-}$.

○ Define a memoryless strategy $\sigma_{\hat{p}}$ obtained by arbitrarily modifying $\sigma_{\hat{p}}^{-}$for $v$ if $\mathrm{P}_{v}=\hat{p}$. Consider in $G$, a $\sigma_{\hat{p}^{-}}$compatible play $\rho$ starting in $\mathrm{W}_{\hat{p}}\left(G^{-}\right)$. Then $\rho$ will never visit $v$ otherwise there would be a $\sigma_{\hat{p}}^{-}$-compatible play in $G^{-}$ reaching $v$. So $\rho$ is a play in $G^{-}$and thus winning for Player $\hat{p}$.

○ Define a memoryless strategy $\sigma_{1-\hat{p}}$ by $\sigma_{1-\hat{p}}^{+}$over $V_{1-\hat{p}} \cap \mathrm{W}_{1-\hat{p}}\left(G^{+}\right)$and by $\sigma_{1-\hat{p}}^{-}$over $V_{1-\hat{p}} \backslash \mathrm{W}_{1-\hat{p}}\left(G^{+}\right)$. Consider in $G$, a $\sigma_{1-\hat{p}}$-compatible play $\rho$ starting in $\mathrm{W}_{1-\hat{p}}\left(G^{-}\right)$.

- Either $\rho$ never visits $\mathrm{W}_{1-\hat{p}}\left(G^{+}\right)$.

Then $\rho$ is a $\sigma_{1-\hat{p}}^{-}$-compatible play in $G^{-}$and so winning for Player $1-\hat{p}$.

- Or a suffix of $\rho$ is a $\sigma_{1-\hat{p}^{-}}^{+}$compatible play in $G^{+}$and so $\rho$ is winning for Player $1-\hat{p}$.

Observations. There are two main differences with the proof in [5]. First we pick a single vertex in $G$ for the induction but more importantly $G^{+}$is somewhat a partial unfolding of $G$ while the reduced graphs considered in [5] only consist in making some vertex $w$ absorbing and possibly changing its priority (like $G^{-}$). In particular, the winning sets of $G^{+}$allow to decide the status of $v$ in $G$ while a graph where $w$ has been made absorbing may provide information for the status of any vertex but $w$ (and this is the way it is exploited in [5]). From an algorithmic point of view, the above proof leads to 
a (highly inefficient) recursive algorithm in $O\left(2^{|V|} D\right)$ where $D$ is the outgoing degree of $G$.

\section{Illustration}

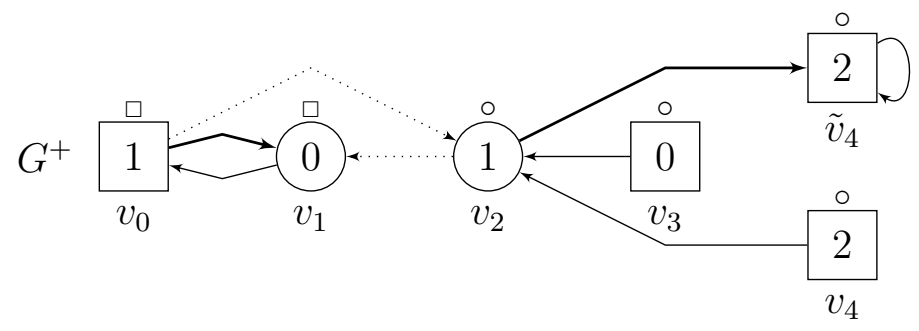

Figure 4: The favourable case.

We illustrate the favourable case (i.e. without use of $G^{-}$) and the unfavourable case on the game of Figure 1. The relevant vertex with maximal priority is $v_{4}$. The corresponding graph $G^{+}$is depicted in Figure 4 obtained by "duplicating" $v_{4}$. Since $v_{4} \in \mathrm{W}_{0}\left(G^{+}\right)$, the strategies for $G^{+}$can be applied to $G$. The thick edges are the ones selected by the strategies; the dotted edges are the discarded ones and the other ones are irrelevant for the winning sets.

Let us apply our proof on the intermediate graph depicted in Figure 4. To obtain its winning sets, one duplicates $v_{2}$ as depicted in Figure 5 producing the graph $\left(G^{+}\right)^{+}$. Since $v_{2} \in \mathrm{W}_{0}\left(\left(G^{+}\right)^{+}\right)$, one needs to build the graph $\left(G^{+}\right)^{-}$ as depicted in Figure 6 . Observe that after solving this game, $v_{3}$ and $v_{4}$ have moved from one winning set to another one.

\section{References}

[1] E. A. Emerson, C. S. Jutla, Tree automata, mu-calculus and determinacy (extended abstract), in: 32nd Annual Symposium on Foundations of Computer Science, San Juan, Puerto Rico, 1-4 October 1991, IEEE Computer Society, 1991, pp. 368-377.

[2] A. W. Mostowski, Games with forbidden positions, Tech. Rep. Technical Report 78, Uniwersytet Gdsuiski, Instytut Matematyki (1991). 


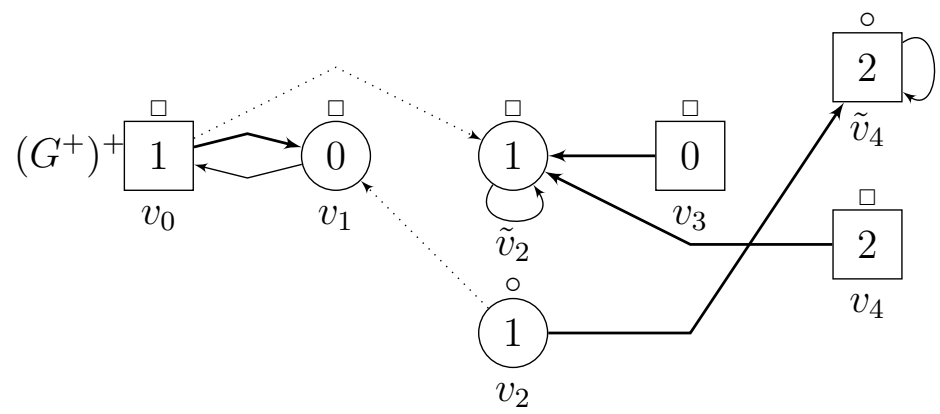

Figure 5: The unfavourable case.

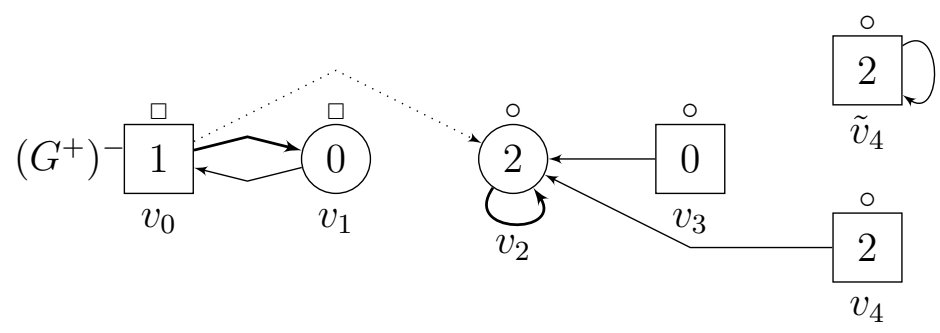

Figure 6: The graph $\left(G^{+}\right)^{-}$.

[3] W. Zielonka, Infinite games on finitely coloured graphs with applications to automata on infinite trees, Theor. Comput. Sci. 200 (1-2) (1998) 135-183.

[4] H. Björklund, S. Sandberg, S. G. Vorobyov, Memoryless determinacy of parity and mean payoff games: a simple proof, Theor. Comput. Sci. 310 (1-3) (2004) 365-378.

[5] E. Grädel, Finite Model Theory and Descriptive Complexity, Springer Berlin Heidelberg, 2007, Ch. 3, pp. 125-230.

[6] B. Karelovic, W. Zielonka, Nearest fixed points and concurrent priority games, in: Fundamentals of Computation Theory - 20th International Symposium, FCT 2015, Gdańsk, Poland, August 17-19, 2015, Proceedings, Vol. 9210 of Lecture Notes in Computer Science, Springer, 2015, pp. 381393. 